\title{
Many to Many Mobile Maps
}

\author{
Stephen C. Hirtle and Martin Raubal
}

\begin{abstract}
The rapid development of mobile computing devices along with a variety of Web 2.0 social networking tools has led to a dramatic change in the way maps and other spatial displays are utilized. The evolution from stand-alone desktop GIS to the interactive, mobile devices, in which information from one or more sources and is sent to one or more sinks, is discussed. The result is access to real-time information, which is generated from both traditional sources, social networks, and other specialized geowikis. Both the benefits of many to many mobile maps and the emergence of new problems, such as understanding the needs of the user and providing appropriate context, are discussed.
\end{abstract}

Keywords Mobile computing • Social computing • GIS

\section{Introduction}

In the past 40 years, Geographic Information (GI), which was once the domain of paper and mechanical tools, has moved into the electronic domain with the development of Geographic Information Systems (GIS). More recently, electronic processing of spatial information has moved from stand-alone desktop systems to interconnected mobile devices. Such evolution in software and hardware has been

\author{
S. C. Hirtle $(\square)$ \\ School of Information Sciences, University of Pittsburgh, Pittsburgh, PA 15260, USA \\ e-mail: hirtle@pitt.edu \\ M. Raubal \\ Institute of Cartography and Geoinformation, ETH Zurich, Zurich, Switzerland \\ e-mail:mraubal@ethz.ch
}


characterized by Frank (2002) as a general shift from a reliance on general purpose geographic information systems (Big GIS) to access of specific geographic information (Small GI), which is relevant for the particular place and time. Big GIS was administered typically by non-end-users, often in a batch mode, for large problems, such as one would find in areas like urban planning, demographic analysis, or topographic mapping. The Small GI approach put the analysis in the hands of individual users and can solve problems that require iterative queries, such as found in logistics, tourism, real estate, or marketing. It is interesting to note that in historical terms, information was in the hands of users before the development of Big GIS. The shift to Small GI has returned spatial information to the hands of individuals.

Overall, the change to Small GI has been transformative and requires a reassessment of the field of geographic information science. Twenty years ago, every day spatial problems, such as determining the directions to a vacation resort or identifying a restaurant for dinner, were often solved in advance using static technologies including paper maps and guides. The use of electronic data was reserved for complex spatial problems, such as tracking the movement of a hurricane or locating the ideal location of a new store.

The shift to the use of spatially aware personal digital assistants over the last two decades has led to a change of the information-seeking behavior and created novel spatio-temporal decision situations due to the dynamic nature of mobility. This change has resulted in a shift of information needs: we often need to make decisions on the spot, for example, locating needed roadside services when traveling by car or making a flight connection inside a busy airport under time pressure. New technologies, such as Location Based Services (LBS), can help individuals make well-informed decisions. However, they can only do so if they take into account how people operate in dynamic and often complex situations, what kind of information they require, and how such information can be communicated effectively.

The result of these changes has been the increase of real-time information that can assist decision-making processes and a change to mobile decision-making that reflects current needs and current conditions. Mobile decisions are increasingly less likely to be made on the basis of centralized databases, but more likely to be supported through the Web 2.0 initiatives of social networking and distributed information sources. In terms of the temporal scale, mobile decisions are often made at the start of any actions and can change as the action progresses. Both push and pull technologies are possible, although push technologies have traditionally been viewed with concern for privacy and invasiveness (Raper et al. 2008; Kaasinen 2005).

To capture this new environment, we introduce the term many to many mobile maps, which can be defined as spatial displays that are geographically aware and shown on small portable devices connected to multiple sources and sinks. That is to say, the device can receive information that has been generated by many users or sensors, and can also send information to many users or act as sensor. For example, a GPS-based navigation system might do variable routing based on current traffic 
flows, while at the same time providing its own traffic speed information to update the data servers. The concept of many to many allows for many to one, such as a restaurant recommendation based on a social network of hundreds of reviews, and one to many, such as a single time-trace of a hike that is sent to a large archive of hikes that can be used to generate future recommendations. Furthermore, the maplike representations in many to many mobile maps can vary from a simple arrow indicating to turn here to photorealistic images of the immediate surround (Butz et al. 2001). At present, a two-dimensional graphical map would be the most common display and is typically displayed on small portable devices, such as an Android phone or iPhone. Satellite views, street-level images, and graphical mockups of the local environment (Agrawala et al. 2011) are also possible under the many to many mobile map framework, as well as both larger and small visual displays, sophisticated audio interfaces, or force-fed input devices.

\section{How Has the Field Changed?}

\subsection{Access to Real-Time Information}

Over the last years we have seen a tremendous change in how people access information and also with regard to the currency of such information. Most mobile decision situations require access to up-to-date information, such as in transportation, emergency response, or weather-related applications. We often think of this kind of information being accessed through mobile devices, but it can also include public information. For example, many public transportation systems offer information screens inside vehicles and/or at stops, which show the departure times of connecting buses, trams, and subways (Fig. 1). This information can also be accessed on mobile devices, which would facilitate to adjust trips in transit or plan alternative stops along the way.

Many to many mobile maps are greatly facilitated by the large amount of sensor data that is readily available through public databases. This includes common

Fig. 1 Public screen inside a tram in Zurich, Switzerland showing time estimates for the next three stops and the final destination, as well as connecting lines at the upcoming train station Bahnhof Oerlikon Ost

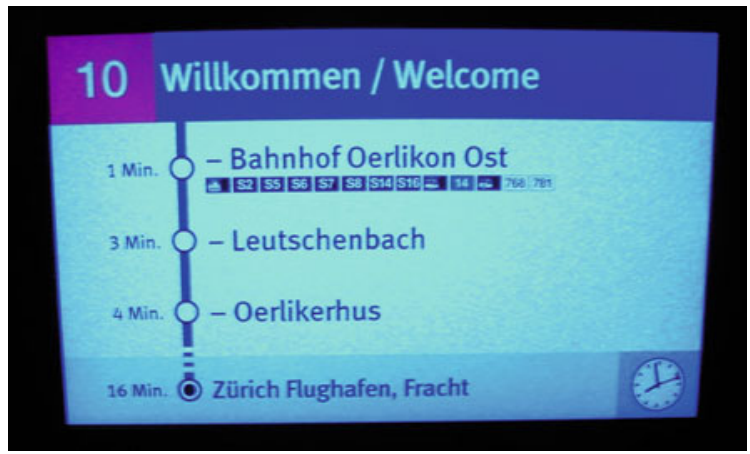


sources such as traffic sensor information or weather data. As just one example of traffic data, the California Department of Transportation maintains over 20,000 inductive loop sensors, which count the number of vehicles that pass over each highway sensor, as well as the time covered by each vehicle, to yield measures of flow and occupancy (Hutchins et al. 2010). Weather data is even more abundant with weather stations reporting a large variety of metaconditions at regular intervals. Efforts, such as the SensorMap project (Nath et al. 2007), provide general portals to both publish and retrieve sensor data. Furthermore, sensor information can be gathered using less traditional means. For example, Nericell (Mohan et al. 2008) is a prototype that uses the capabilities of smartphones, such as the accelerometer, microphone and GPS sensors to record potholes, bumps, braking, and traffic noise in Bangalore, India.

The cloud also allows for the sharing of additional data, computational resources, and specialized analysis programs (Lane et al. 2010). The use of the cloud raises potential privacy issues, especially when your own data is being spread to others without your explicit consent. More common is the ability to share informal sources of information through Twitter, Facebook, Flickr, and other social media, as discussed below, where there is more obvious control over some of the privacy settings through the personal management of your friend networks.

\subsection{Traditional Decision-Making to Mobile Decision-Making}

Clearly, a large part of the world's civilization has turned into a mobile information society, often requiring people to make decisions on the spot and in highly dynamic environments (Raubal 2011). There is still little knowledge about how mobile location-based decision-making is different from other types of decision-making. Much research has been done in the area of general decision theory covering a wide range of models with different foci on describing how decisions could or should be made and on specifying decisions that are made (Golledge and Stimson 1997). Behavioral decision theory has been emphasized in the cognitive literature due to the fact that human decision-making is not strictly optimizing in an economical and mathematical sense (Simon 1955). In order to investigate whether principles of generic decision-making can be transferred to mobile decision-making and find potential differences, researchers have developed tools to study the interaction between environments, individuals, and mobile devices ( $\mathrm{Li}$ and Longley 2006).

Mobile decision-making involves a multitude of spatio-temporal constraints relating not only to people's spatio-temporal behavior in large-scale space (Kuipers and Levitt 1988) but also to their interaction with mobile devices, and perceptual, cognitive, and social processes. Space and time must therefore be considered from a broad perspective and as a context for understanding (Peuquet 2002). This includes actual physical spaces, multiple psychologies of space 
(Montello 1993), and different types of times (Frank 1998). It is obvious that achieving progress in what may be called the evolving field of mobile Geoinformatics, requires multi- and interdisciplinary research. The overarching research question to be tackled is what is special about mobile decision-making?

\subsection{Social Networking}

Social networks have emerged in the decade as a major source of location-based information. Initially, social networking sites were designed both to facilitate communication among groups and to expand one's networks of friendships. Boyd and Ellison (2008) put SixDegrees.com, launched in 1997 and closed in 2000, as the first social network site. SixDegrees was followed by numerous other sites, such as LiveJournal, Friendster, MySpace, Flickr, Facebook, and Twitter. Location information became the focus of some sites, such as FourSquare. Recommendation sites, such as Yelp and Urbanspoon, include geographic pointers, and usergenerated encyclopedias, such as Wikipedia and Wikimapia, include location coordinates where appropriate.

The consequence of the large amount of location-based social networking sites, is that one can gather both real-time information (e.g., the restaurant closed this afternoon due to a water main break) and aggregate information (e.g., travel times on Route 9 are typically 10 miles per hour under the posted limits during the lunch hour). In this rapidly changing arena, the following subsections highlight five different ways in which the social networking is being incorporated into spatial decision making and processing.

\subsubsection{Volunteered Geographic Information}

Volunteered geographic information (VGI) describes a large number of related activities in which collections of individuals provide geographic information for common consumption, in contrast to relying on the traditional authorities alone to provide maps and spatial information (Goodchild 2007). VGI allows individuals to mark information about locations that are of particular interest using either standalone applications or generic platforms. Generic platforms include websites such as OpenStreetMap or Wikimapia. After the devastating 2010 earthquake in Haiti, a group of international volunteers working abroad were able to remotely update OpenStreetMap, indicating passable roads, location of temporary shelters and the like. This was done in innovative ways by using not only current satellite imagery, but also ground reports, video captures and television news reports (Zook et al. 2010). The GeoCommons ${ }^{1}$ project repository provided central storage for a

\footnotetext{
1 http://geocommons.com/
} 
variety of data sets, both official and unofficial. Zook et al. (2010) noted that the repository grew from less than two dozen to over 350 data sets in the weeks after the earthquake.

Other examples are more mundane, but also interesting. For example, the Wikimapia section for Pittsburgh, Pennsylvania indicates the location of the "food trucks," which appear on a daily basis near Carnegie Mellon University to sell food to students (Hirtle 2011). These trucks are part of an informal infrastructure that would not appear in yellow page directories, service listings, or other traditional maps, since they do not reside in permanent buildings with a fixed address. Yet, the information and location of the food trucks is quite useful for students looking for inexpensive meals at lunchtime and, thus, reflects in a GIS the common wisdom of the crowds.

\subsubsection{Specialized Geowikis}

As a subset of VGI, specialized geowikis also provide a platform for the sharing of certain kinds of spatial information. Here, we use the sense of geowiki that was given by Priedhorsky (2011) to refer to a specialized wiki that includes geographic information. ${ }^{2}$ This goes beyond Wikipedia and Wikimapia, which are large-scale, generic repositories of generally unstructured text. As an example of a specialized geowiki, Priedhorsky et al. (2007a, b) demonstrated a bicycle route-finding system, which was based on information collected and stored in a geowiki. This application was challenging, as bicycle navigation systems are particularly difficult to automate. There are strong personal preferences with regard to topography, traffic, distance, and other factors. Priedhorsky et al. (2007a, b) presented a system that allowed for a wide range of personalized comments in terms of both the nature of the route to nearby amenities, such as the location of a pump to get air in your tires to an easy place to stop for refreshments. Building the system required a WYSIWYG web interface that made it easy to add information and bicycle paths. The project also includes a computational component where user-contributed knowledge was fed into selecting optimal routes.

\subsubsection{Space-Time Trails}

More recently, the explosion of GPS-enabled devices on smartphones, mp3 players, cameras, and other small mobile devices has led to the automatic recording and uploading of space-time trails. For example, Every Trail collects space-time trails in the same way that Flickr collects photographs or Delicious

\footnotetext{
2 Our use of the term, geowiki, is in the generic sense of the concept. Geo-Wiki (with a hyphen) is the proper name of a separate project founded in 2009 through a collaboration of the International Institute for Applied Systems Analysis, University of Applied Sciences Wiener Neustadt and the University of Freiburg.
} 
collects bookmarks. Uploaded trails on Every Trail have been categorized by mode of transportation and/or activity; a list which includes road biking, mountain biking, hiking, walking, running, driving, motorcycling, sightseeing, skiing, kayaking, canoeing, sailing, backpacking, roller skating, snowshoeing, horseback riding, snowboarding, ice skating, snowmobiling, hang gliding, skateboarding, bird watching, rock climbing, and even mountain unicycling. This information is publically available and can be easily mined for the collective information about popular routes, accurate estimates of travel times, and other spatio-temporal information. The large number of activities is critical to users, as what makes a good trail for mountain biking, backpacking, or rock climbing is based on a unique set of characteristics and constraints.

One method of aggregating space-time trails is through space syntax (Hillier 1996). Space syntax allows space-time trails to be aggregated across individuals to present the joint conception of space by activities, as shown in Fig. 2, where the time element is collapsed and no longer represented in an explicit fashion. Space syntax then provides a representation of the space by actual use. This might even include anomalies, such as traffic regulations (e.g., one-way streets or stop signs) that are being ignored on a regular basis (Turner 2009). The resulting "map" does not represent the legal truth, but instead the accepted reality of the collective wisdom of the crowd. Thus, space syntax can give a better understanding of the

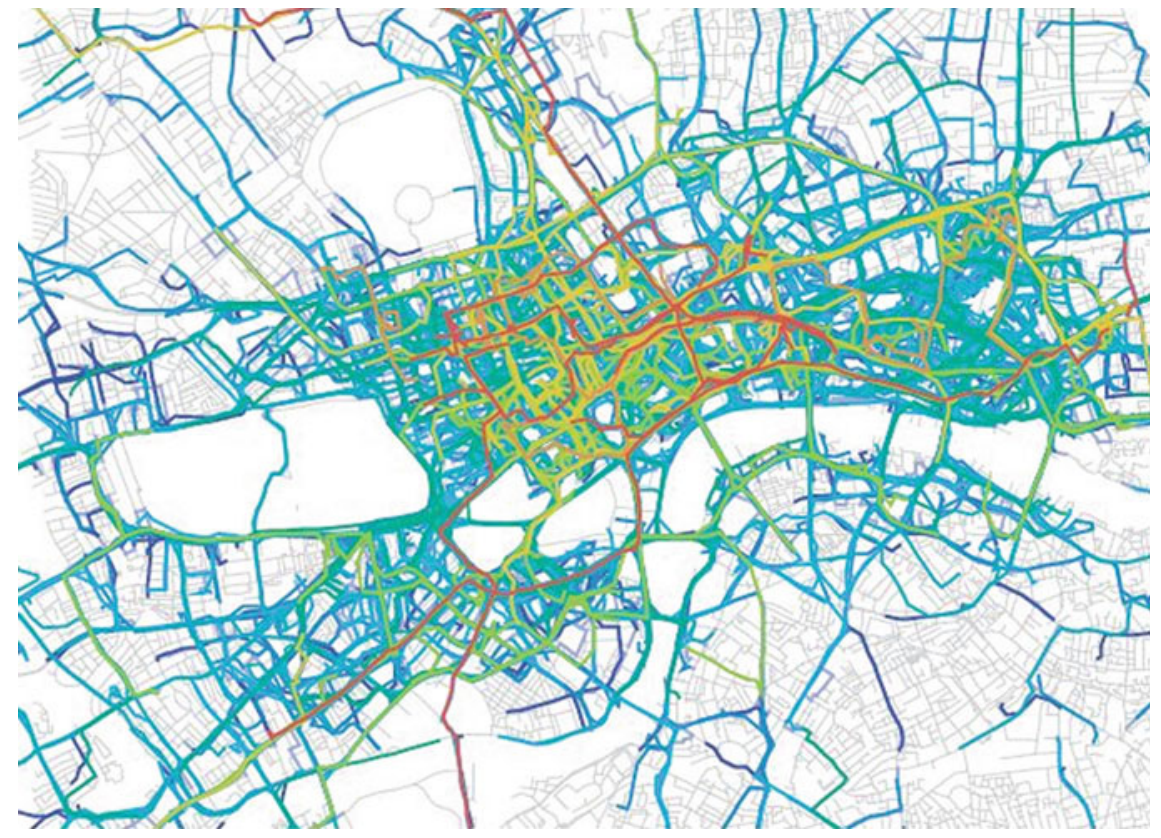

Fig. 2 Space-time trails using space syntax measures from (Turner 2009). The area is from central London covering approximately $8 \mathrm{~km} \times 6 \mathrm{~km}$. Colors indicate trip duration 
structure of a city as perceived by the residents who share their daily travels (Counts and Smith 2007; Turner 2009).

\subsubsection{Place Concepts as Social Constructs}

In addition to examining the information produced through VGI, one can use social tagging in an indirect way to understand the conceptualization that individuals have when referencing locations throughout the city. Social tagging is used as a way of marking entries in large collections (Gupta et al. 2010). The tags can be considered a folksonomy (Gruber 2008), which is not as formal or structured as an ontology, but allows individuals access to the content. The flexibility of social tagging makes it easier for individuals to implement and also allows for new tags to be incorporated into the folksonomy.

Using social tagging in an innovative way to answer deeper questions, Schlieder and Matyas (2009) analyzed a large collection of over 12,000 photographs of cities posted on Panoramio to show how cities are conceptualized by tourists. Schlieder and Matyas (2009) describe the approach as one of collaborative semantics, where the shared locations and terminology can be used to describe the structure of the city. This is not unlike the difference that one finds between the formal structure of a city as determined by city planners and engineers, and the common paths of travel that emerge through an analysis of walking patterns using space syntax (Hillier 1996).

\subsubsection{Social Navigation Services}

A more direct application of social networking can be found in social navigation services (Bilandzic et al. 2008). There is a wide range of services available on the web from location-based recommender systems to virtual post-it notes and graffiti (Espinoza et al. 2001). As with the previous examples, social navigation services can answer questions that require human judgment, such as the best place to find an inexpensive meal, or the best place to get fresh food for a picnic. Many traditional location-based services, such as Yellow Pages, are constructed without regard to quality of service. In contrast most current recommender systems, such as Yelp, reject this view and explicitly include a notion of social recommendation (Hearst 2008; Gupta et al. 2010). 


\section{What are the Challenges and Goals for Future Research?}

\subsection{Interaction Between Environments, Individuals, and Mobile Devices}

In many ways, the ideal mobile device would act like an intelligent tour guide, who is knowledgeable of the immediate surroundings, knows the optimal route to get to a location, adjusts decisions with the current conditions and has intimate knowledge of all the shops, restaurants, and individuals in the area. This Orwellian view of technology in the near future will remain unrealized. Yet, at the same time, many individuals have already relinquished control of decisions to portable devices. We no longer have telephone numbers in memory, but depend on devices to recall them until lost. We now let GPS-based systems plan routes without confirming if the route makes sense in the local environment.

Future research must focus on the interaction possibilities between user, environment, and mobile device. Each of these 'components' can vary and the interaction depends on the specific task (Fig. 3). Different users perceive their environments differently and this should be reflected in the representation of the environment on the mobile device in order to facilitate user interaction. During an exploratory empirical study of interaction differences in the navigation services offered by Apple's iPhone and Google's Android smartphone a significant threeway interaction between the factors device, task, and environment could be demonstrated (Richter et al. 2010). This result implied that the number of wrong turns made by participants depended upon this combination. Further studies including different application scenarios, users, environments, and devices will shed more light on the complexity of this interaction and how to optimize it.

Fig. 3 Task-dependent interaction between agent (user), environment, and representation (mobile device) from (Richter et al. 2010)

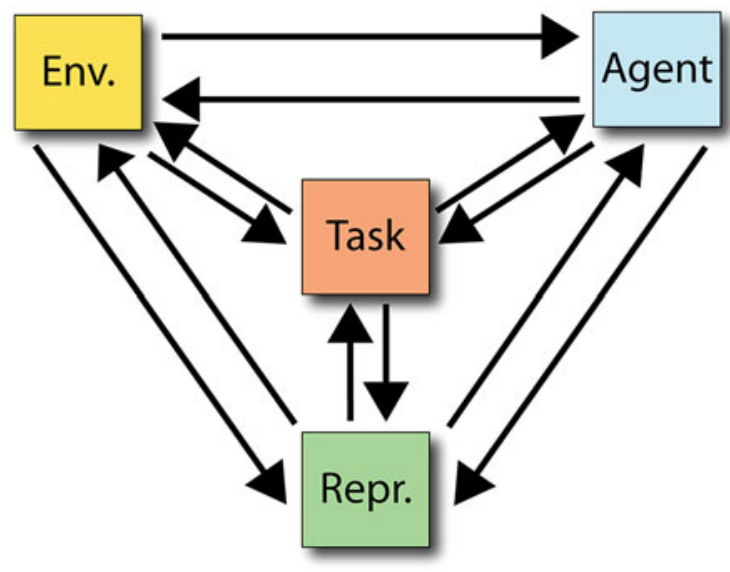


Mobile eye-tracking studies (Grifantini 2010) can help find out whether users focus more on the environment or their mobile devices. The users' movement traces on their mobile device displays should indicate design problems with respect to symbol visualization, instructions, and other task-dependent spatiotemporal information. These studies will be of higher complexity compared to desktop eye-tracking experiments due to additional degrees of freedom (mobile device, movement, etc.).

\subsection{Knowing the User}

A key issue for the development of personalized services is knowing the user. Among the most important variables regarding differences between individual users and user groups (Raubal and Panov 2009) are age, gender, differences in spatial memory and reasoning abilities, preferred learning style, and attitude differences. Mobile decision-making requires fast access to spatial memory and the ability to make quick decisions on the spot. In addition, users have to cope with technological limitations regarding their mobile devices such as small screen size, and there is the general challenge of presenting information to someone on the move. We need studies that investigate for different domains how (different) people actually make decisions while on the move and how these decisions are impacted by technology and its current limitations. Intelligent user interfaces (Maybury and Wahlster 1998), which provide additional benefits to their users, such as adaptivity, context sensitivity, and task assistance, may be the way to go. But here, it is especially important to represent and exploit models of the user, the domain, tasks, and context. Adaptation is also required because it is practically impossible to anticipate the needs and necessities of each potential user in an infinite number of presentation situations.

\subsection{Context}

What is considered an optimal decision strongly depends on the context (Dey and Abowd 2000; Schmidt et al. 1999). Therefore, context elements must be captured for the particular user and task. What are relevant context elements and how can they be formalized (Raubal and Panov 2009)? These days, we have to cope with enormous loads of data, some of them helpful but most of them irrelevant to the task at hand. Much of these data automatically feed from sensors to the mobile device and support the mobile decision-making process. In addition, data can potentially be reused by others, such as when contributed and made available as VGI. Future research will have to address several issues in order to achieve context-sensitive location-based services, such as: How can we assure that only context-relevant data is taken into account? How can we filter these data for a 
particular user? How adaptive is such filtering process to the user's spatial and cognitive capabilities?

\subsection{Impact on Spatial Learning}

The use of technology for supporting people's mobile decision-making does not only impact their task performance but also their spatial learning of the environment. Studies have demonstrated that using mobile navigation devices may result in users turning off their brain (Munzer et al. 2006; Parush et al. 2007). They do not process the presented information and the information perceived in the environment to a sufficient level, and also lack the possibility to acquire survey knowledge. This is critical, especially in situations where technology fails and people must fully rely on their spatial knowledge and abilities.

In a human participants test using a multi-level virtual environment (Parush et al. 2007) demonstrated that reliance on automatic wayfinding systems can result in a degradation of spatial knowledge acquisition and learning. Participants had to perform way finding tasks and their current position was either indicated continuously or by request. In addition, they had to answer sporadic orientation quizzes. The results showed that those participants who had to request their position and who were more 'involved' through orientation quizzes also demonstrated better knowledge acquisition. The real-world study (Richter et al. 2010), described in Sect. 3.1, also indicated that differences in interaction with a mobile device during wayfinding can have an effect on people's spatial learning. More specifically, participants, who were required to actively zoom in at decision points when using a navigation service, made fewer wayfinding errors when later re-walking the same route without the help of the navigation service. Thus, participants showed an improvement in spatial learning with the active engagement of the navigational device.

As just one example of a non-geographic application, recent studies have looked at the role of spatial cognition in surgery and related fields (Hegarty et al. 2007, 2009). With minimally invasive surgical procedures, such as laparoscopy where a miniature video camera is directed to the surgical location, there is a loss of haptic cues that existed with the previous, more invasive forms of surgery. Keehner and Lowe (2009) have shown that haptic cues might reinforce the information gathered from visual cues in surgical settings. Thus, it might be incumbent on future interfaces to include a haptic component for the surgeons to respond. In a very different domain, an experimental interface, which combines auditory feedback and a forcefeel joystick, allows blind sailors to direct a crew on a sailing vessel in tests off the coast of France (Simonnet et al. 2010). Thus, to the extent which spatial interfaces engage the user in the physical environment (Meilinger et al. 2008), there should be additional encoding and better memory of the environment.

Future research should investigate how differences in device design impact both navigation behavior and spatial learning. We have started to investigate how the functionality of a mobile navigation service can be adapted so that everyday users 


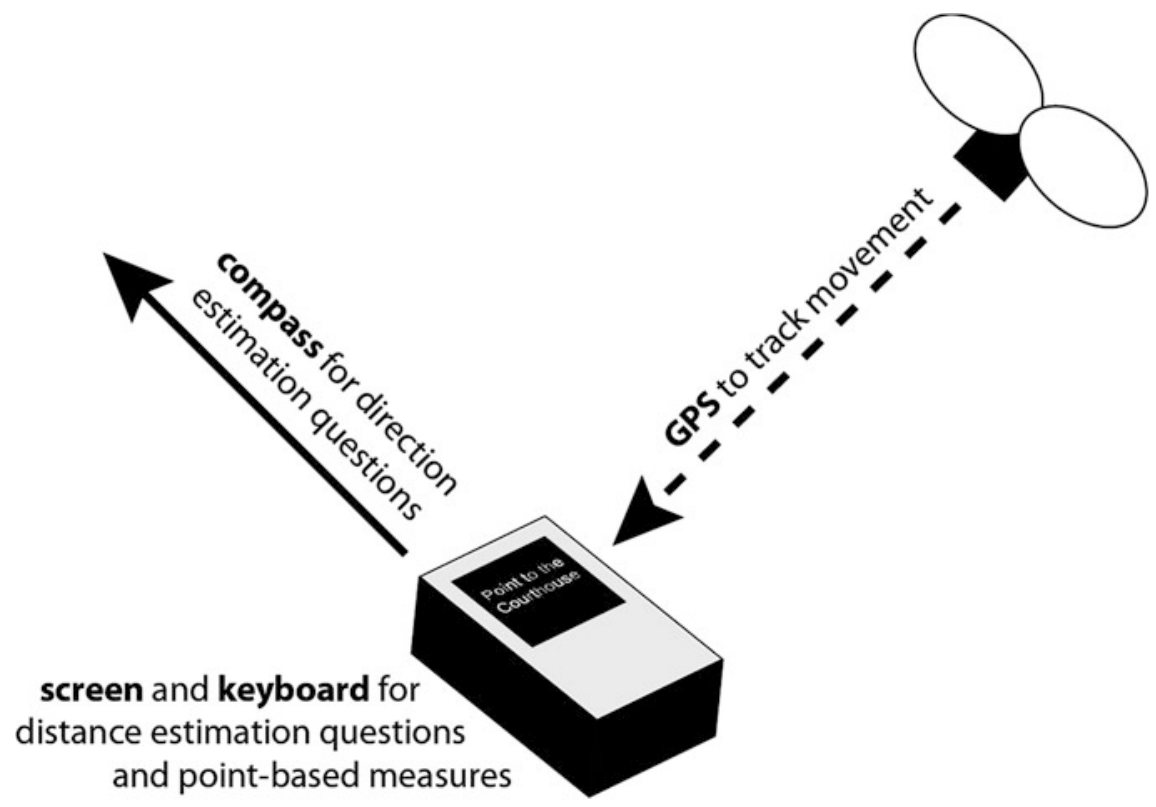

Fig. 4 The cognitive surveyor application supports mobile data collection (Dara-Abrams 2008)

of navigation services achieve both their immediate (finding their goal) and longer-term objectives (spatial learning) (Richter et al. 2010).

\subsection{Innovative Tools}

On the application side, we expect the future to bring novel and pioneering tools and services to the LBS market. Recent research has focused on the integration of digital and analog media (through the use of mobile phones that are equipped with digital cameras) (Rohs et al. 2007), the integration of different modes of communication (such as in navigation devices), and the development of LBS for group-decision-making (Espeter and Raubal 2009). Future mobile guides will be able to access knowledge from diverse online repositories, such as Wikipedia and Wikimapia, and use such content to generate educational audio tours starting and ending at stationary city maps (Schöning et al. 2007).

Innovative tools, such as the Cognitive Surveyor (Dara-Abrams 2008) (Fig. 4) will support future mobile data collection while people are actually performing tasks in the real world. Such data is necessary in order to analyze people's spatial knowledge and navigation practices. It will provide insights on when and where people make decisions, and how their acquired cognitive representations differ from the real world. This will help in the design of better services, which, in turn, should provide enhanced mobile decision support to their users. 


\section{The Future of Maps}

One interesting question that emerges from the preceding analysis concerns the role of maps in the discourse. Consider, for instance, the iBurgh app for the iPhone (McNulty 2009). This application is designed for a single task of reporting a neighborhood problem to the city of Pittsburgh. For example, if you noticed a pothole in your street that has not been filled, you can use the iBurgh app to take a picture of the pothole. The application registers the location and then sends the picture with the geographical coordinates to the city's 311 complaint line. There is no need to describe the location in words or to draw a map of the location to later recall or transmit to the public works department. Spatial information is transmitted directly.

Directions seem like an obvious application for maps. However, maps suffer from the well-studied alignment problem that requires the observer to locate oneself on both the map and in the real world with the appropriate location and orientation (Davies and Peebles 2007). This had led to the needs to include complex instructions to get around the "tricky parts" of verbal directions (Hirtle et al. 2010). Furthermore, (Hirtle and Sorrows 1998) demonstrated that the images can be just as useful as either maps or a spatial description in locating oneself in an environment. A distinctive building would be an easy match in an image, when compared with the task of trying to identify which real-world building corresponds to a colored block representing the building on the map. Agrawala et al. (2011) have explored creating hybrid maps with some 3D sketches of useful landmarks that are of both visual and structural importance (Sorrows and Hirtle 1999).

The influx of image databases, such as Google Streetview, allows one to bypass the mapping process entirely and simply match the target location to an image. In fact, if one needs specific information, such as "Where do I turn to get to my hotel?," one needs no more than a voice telling you when you get near the appropriate intersection to turn in the appropriate direction. At best, this voice is accompanied by a small map. It is curious that on both websites and mobile screens, the $200 \times 200$ pixel map is becoming the norm for locating information.

The downside of providing limited spatial information is that it may impair the acquisition of spatial knowledge. For example, there appears to be a degradation in the ability to acquire spatial knowledge when using guided navigation systems, which results in continued to dependence on guidance systems for repeated trips along the same route (Ishikawa et al. 2008; Parush et al. 2007). The challenge for designers will be to provide effective visual displays that increase a user's engagement with the space, which in turn leads to a greater understanding of the environment (Agrawala et al. 2011). 


\section{Summary}

In this review of literature, we traced a systematic change from centralized GIS platforms to individual GI platforms, which puts information and decision making into the hands of the individual, similar to how GI was viewed during thousands of years of mapmaking. Most of the related research discussed at Las Navas 1990, such as the sense-making investigation method for human wayfinding (Gluck 1991), the evidence that early environmental learning is not necessarily sequential (Blades 1991) as originally proposed by (Siegel and White 1975), or the imageschema-based formalization of interface metaphors by algebraic specifications (Kuhn and Frank 1991)_ _ just to name a few examples_-are still relevant today but the focus has changed due to our mobile information society. Unlike the past, the individual GI platforms are supported by a robust collection of real-time data sources, which can be broadcast to a large array of other users almost instantaneously. Thus, the many to many mobile maps concepts allow for heretofore unprecedented precision in the support of spatial decisions.

\section{References}

Agrawala M, Li W, Berthouzoz F (2011) Design principles for visual communication. Commun ACM 54(4):60-69

Bilandzic M, Foth M, De Luca A (2008) City flocks: designing social navigation for urban mobile information systems. In: 7th ACM conference on designing interactive systems, Cape Town, South Africa, 2008. ACM, New York, pp 174-183

Blades M (1991) Wayfinding theory and research: the need for a new approach. In: Mark D, Frank A (eds) Cognitive and linguistic aspects of geographic space. Kluwer, Dordrecht, pp 137-165

Boyd DM, Ellison NB (2008) Social network sites: definition, history, and scholarship. J Comput Med Commun 13(1):210-230

Butz A, Baus J, Krüger A, Lohse M (2001) A hybrid indoor navigation system. In: IUI2001: international conference on intelligent user interfaces. ACM Press, New York, pp 25-33

Counts S, Smith M (2007) Where were we: communities for sharing space-time trails. In: Proceedings of the 15th annual ACM international symposium on advances in geographic information systems (GIS '07), Seattle, WA, 2007. ACM, New York

Dara-Abrams D (2008) Cognitive surveying: a framework for mobile data collection, analysis, and visualization of spatial knowledge and navigation practices. In: Freksa C, Newcombe N, Gärdenfors P, Wölfl S (eds) Spatial cognition VI. learning, reasoning, and talking about space, LNCS 5248. Springer, Berlin, pp 138-153

Davies C, Peebles D (2007) Strategies for orientation: the role of 3D landmark salience and map alignment. In: Proceedings of the 29th annual conference of the cognitive science society, Nashville, TN, 2007. Erlbaum, New York, pp 923-928

Dey AK, Abowd GD (2000) Towards a better understanding of context and context-awareness. In: Conference on human factors in computing systems (CHI 2000), vol 4. The Hague, The Netherlands

Espeter M, Raubal M (2009) Location-based decision support for user groups. J Locat Based Serv 3(3):165-187 
Espinoza F, Persson P, Sandin A, Nyström H, Bylund ECM (2001) GeoNotes: social and navigational aspects of location-based information systems. In: Abowd BS (ed) Ubicomp 2001: ubiquitous computing, international conference Atlanta, Georgia, September 30October 2. Springer, Berlin, pp 2-17

Frank AU (1998) Different types of "times" in GIS. In: Egenhofer MJ, Golledge RG (eds) Spatial and temporal reasoning in GIS. Oxford University Press, Oxford, pp 40-62

Frank AU (2002) Big GIS/small GI-the commercialization of geographic information. In: Brazilian symposium on geoinformatics, Caxambu, Brazil, 2002

Gluck M (1991) Making sense of human way finding: review of cognitive and linguistic knowledge for personal navigation with a new research direction. In: Mark D, Frank A (eds) Cognitive and linguistic aspects of geographic space. Kluwer, Dordrecht, pp 117-135

Golledge RG, Stimson RJ (1997) Spatial behavior: a geographic perspective. Guilford Press, New York

Goodchild MF (2007) Citizens as sensors: the world of volunteered geography. GeoJ 69(4):211221

Grifantini K (2010) Eye tracking for mobile control. MIT Technol Rev, Cambridge 24 May 2010

Gruber T (2008) Ontology of folksonomy: a mash-up of apples and oranges. Int J Semant Web Inf Syst 3:1-11

Gupta M, Li R, Yin Z, Han J (2010) Survey on social tagging techniques. SIGKDD Explor 12:58-72

Hearst MA (2008) UIs for faceted navigation: recent advances and remaining open problems. In: Workshop on human-computer interaction and information retrieval, Redmond, WA, USA, 2008

Hegarty M, Keehner M, Cohen C, Montello DR, Lippa Y (2007) The role of spatial cognition in medicine: applications for selecting and training professionals. In: Allen GL (ed) Applied spatial cognition: from research to cognitive technology. Erlbaum, Mahwah, pp 285-315

Hegarty M, Keehner M, Khooshabeh P, Montello DR (2009) How spatial abilities enhance, and are enhanced by, dental education. Learn Individ Differ 19(1):61-70

Hillier B (1996) A configurational theory of architecture. Cambridge University Press, Cambridge

Hirtle SC (2011) Geographical design: spatial cognition and geographical information science (synthesis lectures on human-centered informatics). Morgan \& Claypool, San Rafael

Hirtle SC, Richter K-F, Srinivas S, Firth R (2010) This is the tricky part: when directions become difficult. J Sp Inf Sci 1(1):53-73

Hirtle SC, Sorrows ME (1998) Designing a multi-modal tool for locating buildings on a college campus. J Environ Psychol 18(3):265-276

Hutchins J, Ihler A, Smyth P (2010) Probabilistic analysis of a large-scale urban traffic sensor data set. In: Gaber MM, Vatsavai RR, Omitaomu OA, Gama J, Chawla NV, Ganguly AR (eds) Knowledge discovery from sensor data: second international workshop, Sensor-KDD 2008, LNCS 5840. Springer, Berlin, pp 94

Ishikawa T, Fujiwara H, Imai O, Okabe A (2008) Wayfinding with a GPS-based mobile navigation system: a comparison with maps and direct experience. J Environ Psychol 28:7482

Kaasinen E (2005) User acceptance of location-aware mobile guides based on seven field studies. Behav Inf Technol 24:37-49

Keehner M, Lowe R (2009) Seeing with the hands and with the eyes: the contributions of haptic cues to anatomical shape recognition in surgery. In: Bertel S, Barkowsky T, Hölscher C, Shipley TF (eds) Cognitive shape processing. AAAI Press, Menlo Park, pp 8-14

Kuhn W, Frank AU (1991) A formalization of metaphors and image-schemas in user interfaces. In: Mark D, Frank A (eds) Cognitive and linguistic aspects of geographic space. Kluwer, Dordrecht, pp 419-434

Kuipers B, Levitt T (1988) Navigation and mapping in large scale space. Ai Mag 9(2):25-43

Lane ND, Miluzzo E, Lu H, Peebles D, Choudhury T, Campbell AT (2010) A survey of mobile phone sensing. Commun Mag IEEE 48(9):140-150 
Li C, Longley P (2006) A test environment for location-based services applications. Trans GIS 10(1):43-61

Maybury MT, Wahlster W (1998) Readings in intelligent user interfaces. Morgan Kaufmann, San Francisco

McNulty T (2009) iBurgh lets you complain to city by cell phone. Pittsburgh Post-Gazette, Pittsburgh 18 Aug 2009

Meilinger T, Knauff M, Bülthoff HH (2008) Working memory in wayfinding: a dual task experiment in a virtual city. Cogn Sci 32:755-770

Mohan P, Padmanabhan VN, Ramjee R (2008) Nericell: Rich monitoring of road and traffic conditions using mobile smartphones. In: SenSys '08. ACM, Raleigh, North Carolina, pp 323-336

Montello DR (1993) Scale and multiple psychologies of space. In: Frank AU, Campari I (eds) Spatial information theory: a theoretical basis for GIS. Springer, Berlin, pp 312-321

Munzer S, Zimmer HD, Schwalm M, Baus J, Aslan I (2006) Computer-assisted navigation and the acquisition of route and survey knowledge. J Environ Psychol 26(4):300-308

Nath S, Liu J, Zhao F (2007) Sensormap for wide-area sensor webs. Computer 40:90-93

Parush A, Ahuvia S, Erev I (2007) Degradation in spatial knowledge acquisition when using automatic navigation systems. In: Winter S, Duckham M, Kulik L, Kuipers B (eds) Spatial information theory (COSIT 2007) vol LNCS 4736. Springer, Heidelberg, pp 238-254

Peuquet DJ (2002) Representations of space and time. Guilford Press, New York

Priedhorsky R (2011) Wiki, absurd yet successful: a position paper for CHI 2011 workshop on crowdsourcing and human computation. Paper presented at the CHI 2011. Vancouver, BC, Canada, Vancouver, BC, 7-12 May 2011

Priedhorsky R, Jordan B, Terveen L (2007a) How a personalized geowiki can help bicyclists share information more effectively. In Proceedings of the 2007 international symposium on Wikis. ACM, Montreal, Quebec, Canada, pp 93-98)

Priedhorsky R, Jordan B, Terveen L (2007b) How a personalized geowiki can help bicyclists share information more effectively. In: WikiSym'07, Montreal, Quebec, 2007, pp 93-98

Raper J, Gartner G, Karimi H, Rizos C (2008) A critical evaluation of location based services and their potential. J Locat Based Serv 1:4-45

Raubal M (2011) Cogito ergo mobilis sum: the impact of location-based services on our mobile lives. In: Nyerges T, Couclelis H, McMaster R (eds) The SAGE handbook of GIS and society. Sage, Los Angeles, pp 159-173

Raubal M, Panov I (2009) A formal model for mobile map adaptation. In: Gartner G, Rehrl K (eds) Location based services and telecartography II, selected papers from the 5th international symposium on LBS \& Telecartography 2008, Salzburg, Austria. Springer, Berlin, pp 11-34

Richter KF, Dara-Abrams D, Raubal M (2010) Navigating and learning with location based services: a user-centric design. In: Gartner G, Li Y (eds) 7th international symposium on LBS \& teleCartography. Guangzhou, China, pp 261-276

Rohs M, Schöning J, Raubal M, Essl G, Krüger A (2007) Map navigation with mobile devices: virtual versus physical movement with and without visual context. In: Proceedings of the ninth international conference on multimodal interfaces (ICMI 2007). ACM Press, Nagoya, Japan, pp 146-153

Schlieder C, Matyas C (2009) Photographing a city: an analysis of place concepts based on spatial choices. Sp Cogn Comput 9:212-228

Schmidt A, Beigl M, Gellersen HW (1999) There is more to context than location. Comput Graph 23(6):893-901

Schöning J, Hecht B, Rohs M, Starosielski N (2007) WikEar-Automatically generated locationbased audio stories between public city maps. In: Proceedings of Ubicomp'07, pp 128-131

Siegel AW, White SH (1975) The development of spatial representations of large-scale environments. In: Reese HW (ed) Advances in child development and behavior, vol 10. Academic Press, New York

Simon HA (1955) A behavioral model of rational choice. Q J Econ 69(1):99-118 
Simonnet M, Vieilledent S, Jacobson D, Tisseau J (2010) The assessment of non visual maritime cognitive maps of a blind sailor: a case study. J Maps 2010:289-301

Sorrows ME, Hirtle SC (1999) The nature of landmarks for real and electronic spaces. In: Freksa C, Mark D (eds) Spatial information theory. Springer, Berlin, pp 37-50

Turner A (2009) The role of angularity in route choice. In: Hornsby K, Claramunt C, Denis M, Ligozat G (eds) Sp Inf Theory vol LNCS 5756. Springer, Berlin, pp 489-504

Zook M, Graham M, Shelton T, Gorman S (2012) Volunteered geographic information and crowdsourcing disaster relief: a case study of the Haitian earthquake. World Med Health Policy 2(2):7-33 